\title{
Le principe d'accommodement raisonnable en matière religieuse
}

\section{Manon Montpetit et Stéphane Bernatchez}

\section{Q OpenEdition}

1 Journals

Édition électronique

URL : http://journals.openedition.org/rdr/287

DOI : $10.4000 /$ rdr.287

ISSN : 2534-7462

Éditeur

Presses universitaires de Strasbourg

\section{Édition imprimée}

Date de publication : 1 mai 2019

Pagination : 13-40

ISBN : 979-10-344-0045-4

ISSN : 2493-8637

\section{Référence électronique}

Manon Montpetit et Stéphane Bernatchez, « Le principe d'accommodement raisonnable en matière religieuse ", Revue du droit des religions [En ligne], 7 | 2019, mis en ligne le 25 novembre 2019, consulté le 19 novembre 2020. URL : http://journals.openedition.org/rdr/287 ; DOI : https://doi.org/10.4000/rdr. 287

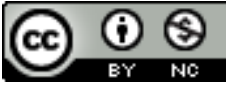

La revue du droit des religions est mise à disposition selon les termes de la Creative Commons Attribution - Pas d'Utilisation Commerciale 4.0 International - CC BY-NC 4.0. 


\section{LEPRINCIPED'ACCOMMODEMENT RAISONNABLEENMATIËRERELIGIEUSE}

\section{Manon MONTPETIT* et Stéphane BERNATCHEZ}

Commission des droits de la personne et des droits de la jeunesse;

Centre de recherche Société, Droit et Religions de I'Université de Sherbrooke (SoDRUS)

\section{RÉSUMÉ}

À partir des principes dégagés par la jurisprudence de la Cour suprême du Canada, le présent article examine les fondements juridiques de l'obligation d'accommodement raisonnable sans contrainte excessive. Cette notion a été développée en vertu du droit à l'égalité protégé par la charte constitutionnelle (la Charte canadienne des droits et libertés) et par la charte quasi-constitutionnelle québécoise (la Charte des droits et libertés de la personne). L'article retrace les principales étapes de cette évolution jurisprudentielle avant de traiter du récent encadrement législatif de l'accommodement raisonnable en matière religieuse.

\section{Abstract}

Based on the principles identified by the Supreme Court of Canada's jurisprudence, this article analyzes the legal basis of the duty of reasonable accommodation without undue hardship. This notion was developed according to the right to equality protected by the constitutional Charter (the Canadian Charter of Rights and Freedoms) and by the quasi-constitutional Quebec Charter (the Charter of Human Rights and Freedoms). This article outlines the main stages of this evolution before dealing with the recent legislative regulation of reasonable accommodation in religious matters specifically.

* Ce texte a été rédigé exclusivement à titre personnel. 


\section{INTRODUCTION}

L'obligation d'accommodement raisonnable, en tant que corollaire du droit à l'égalité, est une notion qui a été essentiellement développée par la jurisprudence afin d'assurer la mise en œuvre d'une "égalité réelle» plutôt que «formelle». Placés devant le dilemme que posent les différentes conceptions - philosophiques et sociales - de ce que constitue l'égalité de traitement, les tribunaux, par une interprétation dynamique des textes de loi, ont ainsi $\mathrm{pu}$ donner tout son sens à un droit, par essence abstrait, mais qui prend assise sur des valeurs de dignité ${ }^{1}$, de partage, d'équité et de tolérance.

Ainsi, s'écartant d'une conception formelle de l'égalité en reconnaissant qu'un traitement identique pour tous pouvait créer des inégalités en fonction de différentes caractéristiques personnelles protégées par la législation sur les droits de la personne, notamment en matière de religion (croyances ou pratiques religieuses), les tribunaux ont accepté le fait que non seulement les différences de traitement pouvaient provenir de préjugés défavorables, mais que des normes et des pratiques, en apparence neutres, pouvaient requérir un traitement différentiel afin de contrer les effets préjudiciables de celles-ci sur certaines personnes. Il s'agissait là d'une avancée importante dans la compréhension des mécanismes et des phénomènes discriminatoires. Par conséquent, l'obligation d'accommodement raisonnable s'est imposée en tant qu'obligation corollaire à la mise en œuvre réelle du droit à l'égalité, sans discrimination. Cette obligation consiste à prévoir l'aménagement d'un traitement différent et adapté pour une personne ${ }^{2}$, afin que l'application de la norme, à son égard, ne puisse compromettre la jouissance pleine et entière de ce droit. Cela signifie aussi que l'on doit toujours tenir compte, dans l'adoption ou l'application d'une norme, des membres de groupes ayant une ou des caractéristiques personnelles protégées par la législation sur les droits de la personne $e^{3}$, ce qui inclut les convictions et les pratiques religieuses.

1. Déclaration universelle des droits de l'homme, art. $1^{\text {er }}$ : "Tous les êtres humains naissent libres et égaux en dignité et en droits. Ils sont doués de raison et de conscience et doivent agir les uns envers les autres dans un esprit de fraternité.»

2. Ou un groupe de personnes.

3. Au Canada, toutes les législatures provinciales ou territoriales et fédérale ont adopté des lois ou des codes visant la protection contre la discrimination fondée sur divers motifs tels, l'âge, le handicap, l'origine ethnique ou nationale, la race, la couleur, le sexe, l'orientation sexuelle, l'identité ou l'expression de genre, le handicap ou le moyen pour pallier le handicap, l'état civil, la condition sociale, la situation familiale. Compte tenu de la Constitution canadienne et du partage des pouvoirs, une loi antidiscriminatoire a aussi été adoptée au 
Les droits des uns devant s'exercer dans le respect du droit d'autrui et de l'intérêt public, les tribunaux ont en outre assujetti cette obligation au critère de raisonnabilité. Ainsi, l'obligation consiste à assurer un traitement favorisant l'inclusion, en toute égalité, sous réserve de la démonstration d'une contrainte excessive pouvant résulter de cette obligation.

Matérialiser en termes juridiques cette conception philosophique du droit à l'égalité en matière de religion est une chose, mais encore faut-il s'assurer de son application dans le monde vécu et le tissu social: le monde du travail, l'administration publique, les contrats, les services offerts au public. Ainsi comprise, la norme d'égalité vient donc remettre en question, dans une certaine mesure, la norme dominante d'égalité formelle. La formulation judiciaire des paramètres de l'accommodement raisonnable, inévitablement, déstabilise les normes établies ${ }^{4}$ et donne ouverture à d'autres remises en question. Depuis plusieurs années, au Canada comme au Québec, les accommodements raisonnables en matière religieuse ont fait l'objet de débats dans les sphères privée et publique, tant sur le plan juridique ${ }^{5}$ que social et politique. Ayant d'abord été une question conceptuelle associée au droit à l'égalité, issue d'une construction juridique jurisprudentielle relativement confinée aux spécialistes en cette matière, la notion d'accommodement raisonnable a été reprise dans le langage populaire ${ }^{6}$, provoquant du coup une certaine incompréhension du concept avec tous les glissements de sens ${ }^{7}$ pouvant en découler.

$\mathrm{Au}$ Québec, le législateur a récemment tenté de concilier le principe de neutralité de l'État en matière religieuse et le respect des valeurs consacrées par la société avec la protection contre la discrimination fondée sur la religion. La courte histoire en cette matière démontre que ces tentatives ont été jusqu'à maintenant difficiles et laborieuses, échouant même dans leurs

niveau fédéral. L'obligation d'accommodement ne s'applique donc pas seulement en matière religieuse mais à tous les motifs de discrimination prévus par ces lois.

4. M. GARON et P. BOSSET, «Le droit à l'égalité: des progrès indéniables, des inégalités persistantes", in La Charte québécoise des droits et libertés après 25 ans, Montréal, Commission des droits de la personne et des droits de la jeunesse, 2003, p. 64.

5. J. Woenrling, «L'obligation d'accommodement raisonnable et l'adaptation de la société à la diversité religieuse», R.D. McGill, 43, 1998, p. 325-401.

6. P. Bosset, «Les fondements juridiques et l'évolution de l'obligation d'accommodement raisonnable», in M. JEZEQUEL (dir.), Les accommodements raisonnables: quoi, comment, jusqu'où? Des outils pour tous, Cowansville, Y. Blais, 2007, p. 6.

7. M. Rivet, «L'accommodement raisonnable ou le nécessaire retour aux sources: le droit à l'égalité», in M. JezEQUel (dir.), op.cit., p. 377: «[...] cette notion, dont l'origine est essentiellement juridique, s'est vue attribuer une nouvelle dimension, portée par l'opinion publique et la confusion qui entoure non seulement les demandes qui sont faites aux différentes institutions, mais les réponses que celles-ci leur donnent.» 
objectifs visant à favoriser une meilleure compréhension de l'état du droit en matière d'accommodement religieux.

Ce texte se veut faire la synthèse, d'un point de vue normatif, du concept d'accommodement raisonnable, d'abord en abordant ses fondements législatifs et jurisprudentiels (1). Sans en faire une analyse exhaustive, nous traiterons les différents cas de figure présentés devant les tribunaux et les problématiques conceptuelles du droit à l'égalité avec lesquelles les décideurs ont dû jongler en matière d'accommodement religieux (2). Enfin, nous examinerons à travers quelques illustrations comment le politique et le législatif ont tenté de répondre aux pressions sociales et juridiques en cette matière (3).

\section{LES FONDEMENTS LÉGISLATIFS ET JURISPRUDENTIELS : LIBERTÉ DE RELIGION ET DROIT À L'ÉGALITÉ}

L'enchâssement du droit à la liberté de religion et du droit à l'égalité contenus dans la Charte canadienne des droits et libertés ${ }^{8}$ et la Charte des

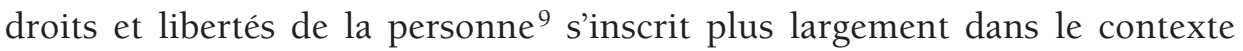
du développement des droits de la personne au niveau international. Avant d'aborder le droit à la liberté de religion ainsi que le droit à l'égalité sans discrimination fondée sur la religion prévus par ces textes constitutionnel et quasi constitutionnel, il convient d'abord d'examiner quelles sont les dispositions pertinentes contenues dans les instruments internationaux similaires.

\subsection{LE DROIT INTERNATIONAL EN TANT QUE SOURCE INTERPRÉTATIVE}

Bien qu'en droit interne canadien les textes internationaux ne peuvent formellement s'imposer dans l'ordre juridique, il demeure que ceux-ci imposent à tous les États parties une reddition de comptes concernant la mise en œuvre effective des dispositions prévues dans les textes internationaux dans leur droit interne par la préparation de rapports périodiques ${ }^{10}$. Ceux-ci font état des mesures adoptées afin de favoriser la mise en œuvre des protections que les États parties se sont engagés à faire respecter; ils confirment en quelque

8. Charte canadienne des droits et libertés, partie I de la Loi constitutionnelle de 1982 [annexe B du Canada Act, 1982 (1982, R.-U., c. 11)] (ci-après «Charte canadienne»). Liberté de religion prévue à l'alinéa 2 a) et droit à l'égalité prévu au paragraphe 15(1).

9. Charte des droits et libertés de la personne, RLRQ, c. C-12. (ci-après «Charte québécoise»). Liberté de religion prévue à l'article 3 et droit à l'égalité prévu à l'article 10 et suivants.

10. De façon périodique. 
sorte les obligations morales des États parties sur la scène internationale. Sur le plan juridique, l'application de ces normes internationales en droit interne a fait l'objet de nombreux questionnements quant à la légitimité des juges à recourir à ces normes lorsqu'elles n'ont pas fait l'objet d'une incorporation explicite en droit interne.

En matière de liberté de religion, la pertinence de l'article 18 du Pacte et des déclarations ${ }^{11}$ afin d'interpréter la liberté de religion prévue à la Charte canadienne a été reconnue à plusieurs reprises dans la jurisprudence canadienne, notamment par la Cour suprême du Canada ${ }^{12}$. Au Québec, le Tribunal des droits de la personne, tribunal spécialisé en matière de discrimination, a particulièrement puisé aux sources du droit international ${ }^{13}$ ainsi qu'au droit des instances étrangères, notamment de la Cour européenne des droits de l'homme, en tant que sources interprétatives des dispositions prévues par la Charte québécoise.

\subsubsection{LA LIBERTÉ DE RELIGION ET DE CONSCIENCE}

Le Pacte international relatif aux droits civils et politiques, ratifié par le Canada et auquel le Québec s'est déclaré lié comporte une protection relative à la liberté de pensée, de conscience et de religion. Son article 18 prévoit que le droit à la liberté de religion - ainsi qu'à la liberté de conscience et de pensée - implique non seulement le droit d'adopter une religion ou une conviction de son choix, mais aussi le droit de manifester celles-ci par le culte, l'accomplissement de rites, les pratiques ou l'enseignement, et ce, individuellement ou en groupe, en privé ou en public. Cette protection implique aussi le droit de ne pas subir de contrainte pouvant porter atteinte à cette liberté, sous réserve «des seules restrictions prévues par la loi et qui sont

11. Déclaration universelle des droits de l'homme; Déclaration sur l'élimination de toutes les formes d'intolérance et de discrimination fondées sur la religion ou la conviction.

12. R. c. Big M Drug Mart Ltd., [1985] 1 R.C.S. 295, 1985 CSC 69, par. 26-27; Chamberlain c. Surrey School District No. 36, [2002] 4 RCS 710, 2002 CSC 86, par. 109; École secondaire Loyola c. Québec (Procureur général), [2015] l RCS 613, 2015 CSC 12, par. 96; Ktunaxa Nation c. ColombieBritannique (Forests, Lands and Natural Resource Operations), [2017] 2 RCS 386, 2017 CSC 54, par. 64-65.

13. Québec (Commission des droits de la personne) c. Autobus Legault, 1994 CanLII 10720 (QC TDP); Commission des droits de la personne et des droits de la jeunesse (Dubé et autre) c. Martin, 1997 CanLII 39 (QC TDP); Commission des droits de la personne et des droits de la jeunesse (Payette) c. Laval (Ville de), 2006 QCTDP 17 (CanLII), par. 94 à 107; Commission des droits de la personne et des droits de la jeunesse c. Centre à la petite enfance Gros Bec, 2008 QCTDP 14 (CanLII), par. 73 à 83; Commission des droits de la personne et des droits de la jeunesse c. Abdelkader, 2012 QCTDP 17 (CanLII), par. 59-60. 
nécessaires à la protection de la sécurité, de l'ordre ou de la santé publique, ou de la morale ou des libertés et droits fondamentaux d'autrui ». Enfin y est prévue une protection relative aux droits des parents ou des tuteurs légaux d'assurer l'éducation religieuse et morale de leurs enfants en conformité avec leurs propres convictions.

Afin de fournir des orientations plus spécifiques aux États parties au Pacte, le Comité des droits de l'homme des Nations unies a adopté l'Observation générale $\mathrm{n}^{\circ} 22^{14}$ relativement au droit à la liberté de conscience et de religion énoncé à l'article 18 du Pacte. Les observations générales du Comité constituent en quelque sorte des sources connexes du droit international; elles prennent toute leur importance en ce qu'elles précisent la portée et la teneur des droits inscrits au Pacte. Quant à l'Observation générale $\mathrm{n}^{\circ} 22$, le Comité y précise que le droit à la liberté de religion prévu à l'article 18 implique celui d'avoir des croyances théistes, non-théistes ou athées, aussi bien que le droit de ne professer aucune religion ou croyance. De plus, le Comité détermine que cette disposition ne se limite pas à la protection des religions ou croyances traditionnelles ou dominantes, mais aussi de celles nouvellement établies. Le droit à la liberté de religion et de conscience implique aussi que personne ne doit être contraint de révéler ses pensées ou d'adhérer à une religion ou à une croyance et que le fait pour un État de reconnaître une religion en tant que religion officielle ne peut avoir pour effet d'entraîner toute forme de discrimination à l'endroit de ceux qui n'y adhèrent pas.

\subsubsection{L'INTERDICTION DE DISCRIMINATION FONDÉE SUR LA RELIGION}

Les articles 2 et 26 du Pacte traitent plus précisément de l'interdiction de la discrimination, notamment en matière de religion. Ces protections visent la protection des droits reconnus dans le Pacte ainsi que l'égale et efficace protection de la loi contre toute discrimination fondée, notamment, sur la religion. Alors que l'article 2 prévoit que les droits qui doivent être protégés contre la discrimination sont limités aux droits énoncés dans le Pacte, l'article 26 ne précise pas une telle limite; il s'agit donc d'un droit autonome.

Puisque le Pacte ne contient pas de définition du terme «discrimination», le Comité des droits de l'homme a aussi adopté l'Observation générale $n^{\circ} 18^{15}$

14. Observation générale $\mathrm{n}^{\circ} 22$, Droit à la liberté de pensées, de conscience et de religion (art. 18), 27 sept. 1993, CCPR/C/21/Rev.1/Add.4.

15. Observation générale ${ }^{\circ} 18$, Non-discrimination (art. 26), 10 nov. 1989, HRI/GEN/1/ Rev.9 (Vol. I). 
portant sur la non-discrimination afin de préciser certaines obligations découlant de ce droit et afin d'en circonscrire le sens. Le Comité considère que le terme «discrimination» doit être compris comme s'entendant de toute distinction, exclusion, restriction ou préférence fondées, notamment, sur un des motifs protégés par le Pacte, dont la religion, et «ayant pour effet ou pour but de compromettre ou de détruire la reconnaissance, la jouissance ou l'exercice par tous, dans des conditions d'égalité, de l'ensemble des droits de l'homme et des libertés fondamentales ${ }^{16} »$. Ce droit n'étant pas absolu, le Comité prend soin de souligner que «toute différenciation ne constitue pas une discrimination, si elle est fondée sur des critères raisonnables et objectifs et si le but visé est légitime au regard du Pacte ${ }^{17}$ ».

D'autres instruments internationaux, qui n'ont pas été ratifiés, mais plutôt adoptés par proclamation par l'Assemblée des Nations unies, ont consacré le droit à la liberté de religion et de conscience au sein de leur texte. La Déclaration universelle des droits de l'homme ${ }^{18}$, pour la première fois dans la sphère internationale, a donné une définition de ce droit. Cette déclaration a aussi consacré le droit à la non-discrimination ${ }^{19}$ fondée, notamment, sur la religion. La Déclaration sur l'élimination de toutes les formes d'intolérance et de discrimination fondées sur la religion ou la conviction prévoit plusieurs articles visant plus particulièrement la protection contre la discrimination fondée sur la religion.

\subsection{LE DROIT CONSTITUTIONNEL ET QUASI-CONSTITUTIONNEL EN DROIT CANADIEN}

Présentant des similarités étroites avec les instruments internationaux, tant la Charte canadienne (art. 2a) que la Charte québécoise (art. 3) consacrent le droit à la liberté de religion. La Cour suprême du Canada a indiqué ceci quant au contenu de ces dispositions: «Vu la similitude entre l'art. 3 de la Charte québécoise et l'art. 2 de la Charte canadienne, il est acquis qu'il convient

16. Ibid., par. 7

17. Ibid., par. 13 .

18. Déclaration universelle des droits de l'homme, art. 18: «Toute personne a droit à la liberté de pensée, de conscience et de religion; ce droit implique la liberté de changer de religion ou de conviction ainsi que la liberté de manifester sa religion ou sa conviction, seule ou en commun, tant en public qu'en privé, par l'enseignement, les pratiques, le culte et l'accomplissement des rites. »

19. Ibid., art. 2: «1. Chacun peut se prévaloir de tous les droits et de toutes les libertés proclamés dans la présente Déclaration, sans distinction aucune, notamment de race, de couleur, de sexe, de langue, de religion, d'opinion politique ou de toute autre opinion, d'origine nationale ou sociale, de fortune, de naissance ou de toute autre situation. [...]» 
d'interpréter la première disposition à la lumière des principes dégagés de l'application de la Charte canadienne ${ }^{20} »$.

La Constitution du Canada, par l'introduction, en 1982, de la Charte canadienne, prévoit expressément la protection de la liberté de conscience et de religion. Ainsi, les actions législatives et gouvernementales doivent respecter et ne pas interférer avec l'exercice de cette liberté fondamentale. Cette protection est toutefois tempérée par la possibilité de recourir d'une part, à une clause dérogatoire (pour certains droits seulement, dont la liberté de religion et le droit à l'égalité) et, d'autre part, à une clause limitative afin de justifier une atteinte à cette liberté fondamentale dans des limites raisonnables, dans le cadre d'une société libre et démocratique. Par ailleurs, aucune disposition expresse de la Constitution ne prévoit la neutralité religieuse de l'État. C'est par développement jurisprudentiel ${ }^{21}$ que les tribunaux canadiens ont reconnu ce principe, lequel est non seulement sous-jacent au respect de l'exercice de la liberté de religion de chacun, mais à l'exercice de cette liberté en toute égalité, sans discrimination.

La Charte canadienne s'applique tant aux lois fédérales que provinciales ${ }^{22}$. Elle s'applique aussi aux actes des paliers gouvernementaux. La Charte canadienne est formellement constitutionnelle puisqu'elle est la «loi suprême» qui peut rendre inopérantes les dispositions incompatibles de toutes autres lois ${ }^{23}$ avec la Charte.

20. Mouvement laïque québécois c. Saguenay (Ville), [2015] 2 RCS 3, 2015 CSC 16, par. 68. De la même manière, dans l'arrêt Syndicat Northcrest c. Amselem, [2004] 2 RCS 551, 2004 CSC 47, par. 37, la Cour suprême du Canada avait indiqué que le contenu du droit à la liberté de religion est le même qu'il s'agisse de la Charte canadienne ou de la Charte québécoise: «Dans l'analyse qui suit, je fais état des principes applicables lorsqu'une personne prétend qu'il a été porté atteinte à la liberté de religion que lui garantit la Charte québécoise ou la Charte canadienne des droits et libertés. »

21. V. Mouvement laïque québécois c. Saguenay (Ville), précit. note 20, par. 71: «Ni la Charte québécoise ni la Charte canadienne n'énoncent explicitement l'obligation de neutralité religieuse de l'État. Cette obligation résulte de l'interprétation évolutive de la liberté de conscience et de religion. [...]» V. aussi dans Congrégation des témoins de Jéhovah de StJérôme-Lafontaine c. Lafontaine (Village), [2004] 2 RCS 650, 2004 CSC 48, par. 67: «Sans faire abstraction des héritages historiques de notre pays, la jurisprudence de notre Cour reconnaît cet aspect de la liberté de religion. Cette conception de la neutralité laisse une place importante aux Églises et à leurs membres dans l'espace public où se déroulent les débats sociaux, mais voit dans l'État un acteur essentiellement neutre dans les rapports entre les diverses confessions et entre celles-ci et la société civile.»

22. V. l'art. 32 de la Loi constitutionnelle de 1982, Annexe B du Canada Act, 1982 (R-U), 1982, c 11 .

23. Ibid., art. 52 


\subsubsection{LE DROIT À LA LIBERTÉ DE CONSCIENCE ET DE RELIGION : PORTÉE ET CONTENU}

La portée du droit à la liberté de religion a été définie par la Cour suprême du Canada comme le droit de croire ce que l'on veut, de professer ses croyances religieuses, par la pratique, par le culte, par l'enseignement ou par la propagation des croyances. De plus, la Cour indiquait que la liberté de religion implique nécessairement le droit de ne pas être contraint d'adopter une certaine conduite religieuse que l'on n'aurait pas choisi d'adopter. Enfin, le droit à la liberté de religion incorpore aussi les manifestations d'incroyance ${ }^{24}$, ainsi que le refus d'observer les pratiques religieuses. Ce droit protège donc «également les intérêts des athées, des agnostiques, des sceptiques et des indifférents ${ }^{25} »$. La Cour indiquait en ces termes:

«Le concept de la liberté de religion se définit essentiellement comme le droit de croire ce que l'on veut en matière religieuse, le droit de professer ouvertement des croyances religieuses sans crainte d'empêchement ou de représailles et le droit de manifester ses croyances religieuses par leur mise en pratique et par le culte ou par leur enseignement et leur propagation. Toutefois, ce concept signifie beaucoup plus que cela.

La liberté peut se caractériser essentiellement par l'absence de coercition ou de contrainte. Si une personne est astreinte par l'État ou par la volonté d'autrui à une conduite que, sans cela, elle n'aurait pas choisi d'adopter, cette personne n'agit pas de son propre gré et on ne peut pas dire qu'elle est vraiment libre ${ }^{26}$.»

Dans l'arrêt Amselem ${ }^{27}$, rendu en 2004, la Cour suprême du Canada a donné à la liberté de religion un contenu juridique très large en indiquant que, dans l'appréciation de la sincérité, les tribunaux doivent « uniquement s'assurer que la croyance religieuse invoquée est avancée de bonne foi, qu'elle n'est ni fictive ni arbitraire et qu'elle ne constitue pas un artifice». Partant, la Cour rejetait l'idée qu'une preuve d'expert soit pertinente puisque les croyances «ont un caractère éminemment personnel et peuvent facilement varier d'une personne à l'autre».

Une fois le fardeau de preuve d'une atteinte à la liberté de religion rencontré, les mécanismes de justifications devront être présentés afin de démontrer que le droit d'une personne à la liberté de religion peut être limité

24. Congrégation des témoins de Jéhovah de St-Jérôme-Lafontaine c. Lafontaine (Village), précit. note 21 , par. 65 .

25. Alberta c. Hutterian Brethren of Wilson Colony, [2009] 2 RCS 567, 2009 CSC 37, par. 90.

26. R. c. Big M Drug Mart Ltd., précit. note 12, par. 94-95.

27. Ibid. 
par d'autres considérations. En matière constitutionnelle, l'analyse devra être menée en fonction de l'article $1^{\text {er }}$ de la Charte canadienne ${ }^{28}$, lequel permet au législateur ou à l'action gouvernementale d'imposer des limites aux libertés et droits fondamentaux. Sous la Charte québécoise, le deuxième alinéa de l'article $9.1^{29}$ joue le même rôle ${ }^{30}$, alors que son premier alinéa vient indiquer «la manière d'interpréter l'étendue de ces libertés et droits fondamentaux ${ }^{31}$ » par un exercice de pondération des droits.

\subsubsection{LE DROIT À L'ÉGALITÉ SANS DISCRIMINATION FONDÉE SUR LA RELIGION ET LA NOTION D'ACCOMMODEMENT RAISONNABLE}

\section{L'article 15 de la Charte canadienne}

Le droit à l'égalité prévu à l'article $15^{32}$ se retrouve, dans la hiérarchie des normes, au sommet de la pyramide, comme tous les autres droits et libertés de la Charte canadienne. Toutefois, bien que la Cour suprême du Canada ait reconnu à travers sa jurisprudence que le paragraphe 15 (1) de la Charte canadienne visait à favoriser l'égalité réelle ${ }^{33}$, et que c'est l'effet réel d'une mesure sur un individu qui doit déterminer si cette mesure est discriminatoire, l'examen constitutionnel de la législation ou des actes gouvernementaux en vertu du droit à l'égalité prévu au paragraphe 15 (1) se prête mal à un

28. «1. La Charte canadienne des droits et libertés garantit les droits et libertés qui y sont énoncés. Ils ne peuvent être restreints que par une règle de droit, dans des limites qui soient raisonnables et dont la justification puisse se démontrer dans le cadre d'une société libre et démocratique.»

29. «9.1 Les libertés et droits fondamentaux s'exercent dans le respect des valeurs démocratiques, de l'ordre public et du bien-être général des citoyens du Québec. La loi peut, à cet égard, en fixer la portée et en aménager l'exercice.»

30. V. Mouvement laïque québécois c. Saguenay (Ville), précit. note 20, par. 90 : «Cet article 9.1 offre à l'État la possibilité de démontrer que la disposition dont les effets attentent à la liberté de conscience et de religion d'un individu constitue une limite raisonnable et justifiée à cette liberté dans le cadre d'une société libre et démocratique. [...]»

31. Ford c. Québec (Procureur général), [1988] 2 RCS 712, 1988 CSC 19, par. 63.

32. Entré en vigueur en 1985 seulement. V. art 32 (3) de la Loi constitutionnelle de 1982. Le par. 1 de l'art. 15 de la Charte canadienne prévoit un droit à l'égalité autonome libellé comme suit: «15 (1) La loi ne fait acception de personne et s'applique également à tous, et tous ont droit à la même protection et au même bénéfice de la loi, indépendamment de toute discrimination, notamment des discriminations fondées sur la race, l'origine nationale ou ethnique, la couleur, la religion, le sexe, l'âge ou les déficiences mentales ou physiques.» Il s'agit d'un droit autonome par rapport aux autres droits qui prescrit une interdiction générale de discrimination dans l'exercice des droits prévus par la loi.

33. Québec (Procureur général) c. A, 2013 CSC 5; Withler c. Canada (Procureur général), 2011 CSC 12. 
examen individualisé des réparations ${ }^{34}$, en cas d'incompatibilité. Par conséquent, la Constitution ne trouvant application qu'envers l'État, tant dans le domaine fédéral que provincial, c'est principalement à travers la législation canadienne en matière de droits de la personne ${ }^{35}$, laquelle s'applique non seulement à l'État mais aux individus entre eux, que la mise en œuvre de l'obligation d'accommodement en matière religieuse a pu se développer et se concrétiser au cours des dernières décennies.

\section{La Charte québécoise et la législation sur les droits de la personne en matière de droit à l'égalité, sans discrimination}

Contrairement à la Charte canadienne, qui a été enchâssée dans la Constitution canadienne en 1982, la Charte québécoise ne possède pas de statut constitutionnel. Toutefois, la jurisprudence canadienne a considéré qu'une telle loi, tout comme les législations de même nature au Canada, possède un caractère spécial, allant même jusqu'à qualifier cet instrument de quasi constitutionnel ${ }^{36}$.

La notion d'accommodement raisonnable en matière religieuse tire sa source de la mise en œuvre réelle du droit à l'égalité. Au Canada, comme au Québec, c'est la législation en matière de droits de la personne plutôt que la Constitution canadienne qui a permis l'adoption de la notion d'accommodement raisonnable comme corollaire du droit à l'égalité. C'est d'ailleurs en s'inspirant de la définition de la discrimination développée par la jurisprudence portant sur la législation canadienne en matière de protection contre la discrimination que la notion de discrimination en matière constitutionnelle

34. Bien que l'exemption constitutionnelle, en tant que réparation, puisse avoir une certaine similitude avec la notion d'accommodement raisonnable appliqué à une personne, la Cour suprême n'a pas formellement intégré cette notion dans le cadre de son analyse du droit à l'égalité en matière constitutionnelle, V. j. Lamer dans Rodriguez c. Colombie-Britannique (Procureur général), [1993] 3 RCS 519, 1993 CSC 75, p. 549. La Cour suprême semble toutefois avoir appliqué cette notion, par analogie, dans l'examen de l'atteinte minimale du test de Oakes, dans l'arrêt Multani c. Commission scolaire Marguerite Bourgeoys, [2006] 1 R.C.S. 256, 2006 CSC 6, la Cour ayant disposé du pourvoi en vertu de la liberté de religion (art. 2 a) de la Charte canadienne), plutôt que sous l'angle de la discrimination fondée sur la religion. V. par. 53. La Cour suprême a abandonné cette approche dans Alberta c. Hutterian Brethren of Wilson Colony, précit. note 25, par. 70: "Plutôt que d'essayer d'adapter la notion de "contrainte excessive" au contexte de l'article premier de la Charte, il est préférable de parler d'atteinte minimale et de proportionnalité des effets.» V. aussi Doré c. Barreau du Québec, [2012] 1 RCS 395, 2012 CSC 12, par. 36, lorsqu'il s'agit d'appliquer les valeurs prévues par les chartes dans les décisions administratives.

35. Toutes les législatures provinciales ou territoriales et fédérale ont adopté des lois ou des codes visant la protection contre la discrimination.

36. Québec (Commission des droits de la personne et des droits de la jeunesse) c. Montréal (Ville); Québec (Commission des droits de la personne et des droits de la jeunesse) c. Boisbriand (Ville), [2000] 1 RCS 665, 2000 CSC 27, par. 27. 
a été appliquée dans le premier arrêt de la Cour suprême ${ }^{37}$ rendu en vertu du paragraphe 15 (1) de la Charte canadienne.

L'article 10 de la Charte québécoise établit en tout premier article du chapitre I.1 ce que signifie la notion de discrimination. Il prévoit ce qui suit:

«Toute personne a droit à la reconnaissance et à l'exercice, en pleine égalité, des droits et libertés de la personne, sans distinction, exclusion ou préférence fondée sur la race, la couleur, le sexe, la grossesse, l'orientation sexuelle, l'état civil, l'âge sauf dans la mesure prévue par la loi, la religion, les convictions politiques, la langue, l'origine ethnique ou nationale, la condition sociale, le handicap ou l'utilisation d'un moyen pour pallier ce handicap.

Il y a discrimination lorsqu'une telle distinction, exclusion ou préférence a pour effet de détruire ou de compromettre ce droit.»

Le chapitre dans lequel l'article 10 s'inscrit s'intitule «Droit à l'égalité dans la reconnaissance et l'exercice des droits et libertés». Contrairement à la protection constitutionnelle prévue au paragraphe 15 (1) de la Charte canadienne, la terminologie employée à l'article 10 de la Charte québécoise sous-tend que la norme d'égalité ne possède pas, en soi, un caractère indépendant ${ }^{38}$, celle-ci indiquant plutôt comme condition qu'une atteinte au droit à l'égalité, sans discrimination, doive porter sur l'exercice d'une liberté ou d'un droit protégé par la Charte. La jouissance de tout droit prévu par la loi n'est donc pas assurée. L'article 10 a donc la même portée limitée que l'article 14 de la Convention européenne des droits de l'homme ${ }^{39}$.

37. Andrews c. Law Society of British Columbia, [1989] 1 RCS 143, 1989 CSC 2, p. 170 : «Que signifie le terme discrimination? C'est le plus souvent dans l'examen des lois sur les droits de la personne que cette question s'est posée et le concept général de discrimination en vertu de ces lois a été assez bien circonscrit. Vu les arrêts rendus par cette Cour, identifier une définition acceptable présente peu de difficulté.»

38. V. Gosselin c. Québec (Procureur général), [2002] 4 RCS 429, 2002 CSC 84, par. 430: "Cette symbiose entre l'art. 10 et les autres droits et libertés découle directement de la formulation de l'art. 10 qui ne crée pas un droit autonome à l'égalité, mais une modalité de particularisation des divers droits et libertés reconnus (Desroches c. Commission des droits de la personne du Québec, [1997] R.J.Q. 1540 (C.A.), p. 1547). En effet, l'art. 10 proclame le droit à l'égalité, mais uniquement dans la reconnaissance et l'exercice des droits et libertés garantis. Aussi, une personne ne peut fonder un recours sur le droit à l'égalité prévu à l'art. 10 en tant que droit indépendant. Elle peut toutefois jumeler l'art. 10 avec un autre droit ou une autre liberté garanti par la Charte québécoise afin d'obtenir réparation pour une distinction discriminatoire dans la détermination des modalités de ce droit ou de cette liberté.»

39. Toutefois, compte tenu de ce champ d'application limité, l'adoption du Protocole $\mathrm{n}^{\mathrm{o}} 12$ à la Convention est venue ajouter à la protection déjà offerte par l'article 14 dans la mesure où le libellé du premier article de ce Protocole prescrit une interdiction générale de discrimination. 
Par ailleurs, une exclusion ou une distinction fondée sur la religion, sans que le droit à la liberté de religion soit invoqué, pourra fonder un recours lorsque ce droit est exercé dans un des domaines d'activités particuliers que le législateur québécois a voulu protéger aux articles 11 à 18.1 et 19 de la Charte québécoise. L'article 10.1 assure par ailleurs une protection contre tout harcèlement, notamment fondé sur la religion. En matière religieuse, la protection contre la discrimination implique donc une protection contre une distinction, exclusion ou préférence fondée sur le motif interdit de discrimination, "la religion», et qui a pour effet de compromettre l'exercice du droit à l'égalité. C'est cette même conception de la discrimination qui prévaut dans la législation sur les droits de la personne au Canada. La Cour suprême du Canada a indiqué à ce titre que: «Si les lois en matière de droits de la personne doivent être interprétées en fonction de l'objet visé, les différences de formulation entre les provinces ne devraient pas masquer les fins essentiellement semblables de ces dispositions, à moins que la formulation n'indique la poursuite d'une fin différente de la part d'une législature provinciale particulière ${ }^{40}$.»

Bien que la liberté de religion puisse être invoquée afin de fonder un recours en discrimination, et qu'on puisse accepter qu'une personne puisse subir une discrimination dans l'exercice de son droit à la liberté de religion, c'est principalement en vertu d'une distinction fondée sur le motif de discrimination interdit, "la religion ou la croyance», que la jurisprudence en matière d'accommodement raisonnable a été développée ${ }^{41}$ au Canada et non selon une atteinte à la liberté de religion dans l'exercice du droit à l'égalité ${ }^{42}$. Il convient de rappeler que les lois antidiscriminatoires au Canada sont principalement consacrées à la discrimination fondée sur un motif interdit de discrimination, dont la religion, dans l'exercice d'un des domaines d'activités prévus dans ces lois et non pas dans l'exercice d'un droit ou d'une liberté. Ces dernières ne prévoient pas de dispositions analogues à celles de l'article 10 de la Charte québécoise, lequel prévoit l'interdiction de discrimination dans l'exercice et la reconnaissance des droits et libertés. Il en

40. Université de la Colombie-Britannique c. Berg, [1993] 2 R.C.S. 353, 1993 CSC 89, p. 373. V. aussi Nouveau-Brunswick (Commission des droits de la personne) c. Potash Corporation of Saskatchewan Inc., [2008] 2 RCS 604, 2008 CSC 45, par. 68: «Il est également établi que l'interprétation d'une loi sur les droits de la personne doit s'harmoniser avec celle de dispositions comparables dans d'autres ressorts. La formulation des garanties et des exceptions peut varier, mais leur interprétation doit demeurer cohérente, sauf intention contraire manifeste du législateur.»

41. J. Woehrling, art. cit. note 5, p. 328.

42. À de rares exceptions. V. Mouvement laïque québécois c. Saguenay (Ville), précit. note 20. 
ressort que la portée du droit à l'égalité sans discrimination prévu à la Charte québécoise est bien plus large ${ }^{43}$ que celle contenue dans la législation sur les droits de la personne au Canada. Ces lois et ces codes antidiscriminatoires ne protègent donc pas, au plan normatif, l'exercice du droit à la «liberté de religion» sans discrimination.

Cela dit, la portée et le contenu définitionnel de «la liberté de religion et de conscience» en matière constitutionnelle, tel que développés par la Cour suprême du Canada, pourront être utiles lorsqu'il est question de faire entrer en jeu le motif «religion» en tant que motif interdit de discrimination inscrit tant dans la Charte québécoise que dans les autres lois sur les droits de la personne au Canada. La jurisprudence en matière de liberté de religion est donc utile et les tribunaux puisent fréquemment aux sources des enseignements de la Cour suprême en cette matière.

La discrimination en matière religieuse peut être sous forme directe ou indirecte. On peut dire que c'est en raison de la reconnaissance que la discrimination peut tirer sa source des effets préjudiciables qu'une norme en apparence neutre peut produire que la notion d'accommodement raisonnable a été développée par la jurisprudence. Ces développements ont été rendus possibles grâce à l'interprétation large et libérale ${ }^{44}$ que les tribunaux, notamment la Cour suprême du Canada, ont donnée à la législation canadienne sur les droits de la personne, allant même jusqu'à consacrer la nature quasi constitutionnelle de celle-ci.

43. Le droit à l'égalité prévu à l'art. 10 de la Charte québécoise a été interprété par les tribunaux et la doctrine comme ayant une portée analogue à l'art. 14 de la Convention européenne des droits de l'homme.

44. Commission ontarienne des droits de la personne c. Simpsons-Sears, [1985] 2 RCS 536, 1985 CSC 18: «Une loi de ce genre est de nature spéciale. Elle n'est pas vraiment de nature constitutionnelle, mais elle est certainement d'une nature qui sort de l'ordinaire. Il appartient aux tribunaux d'en chercher l'objet et de le mettre en application [...]»; Robichaud c. Canada (Conseil du Trésor), [1987] 2 RCS 84, 1987 CSC 73, par. 8: « [...] conformément à la Loi d'interprétation qui exige que les lois soient interprétées de la façon juste, large et libérale la plus propre à assurer la réalisation de leurs objets »; Gould c. Yukon Order of Pioneers, [1996] 1 RCS 571, 1996 CSC 231, par. 120: «La nature particulière des lois sur les droits de la personne demeure l'axiome sur lequel notre Cour fonde sa méthode d'interprétation de ces lois.» 


\section{LA NOTION D'ACCOMMODEMENT RAISONNABLE EN MATIÈRE RELIGIEUSE : FONDEMENTS JURISPRUDENTIELS RELATIFS À LA LÉGISLATION SUR LES DROITS DE LA PERSONNE}

\subsection{UN PRINCIPE FONDÉ SUR L'OBJET PROTECTEUR DE LA LOI}

C'est dans l'arrêt Simpsons-Sears que la Cour suprême du Canada a d'abord reconnu que des normes en apparence neutres peuvent néanmoins causer de la discrimination par les effets préjudiciables qu'elles entraînent et que par conséquent la «conséquence naturelle de la reconnaissance d'un droit doit être l'acceptation sociale de l'obligation générale de le respecter et de prendre des mesures raisonnables afin de le protéger ${ }^{45} »$.

Dans ce litige, une employée de l'Église adventiste du septième jour ayant un statut permanent au sein de l'entreprise alléguait avoir été victime de discrimination fondée sur la religion en raison d'une condition d'emploi qui l'obligeait à travailler le vendredi soir et le samedi. Il s'agissait pour cette personne d'une condition d'emploi contraire aux prescriptions de sa religion. La Cour suprême du Canada donne raison à l'employée en reconnaissant qu'une règle par ailleurs adoptée honnêtement, sans intention discriminatoire, pouvait avoir des effets préjudiciables en compromettant le droit aux croyances religieuses de la plaignante:

«Ce genre de discrimination se produit lorsqu'un employeur adopte, pour des raisons d'affaires véritables, une règle ou une norme qui est neutre à première vue et qui s'applique également à tous les employés, mais qui a un effet discriminatoire pour un motif prohibé sur un seul employé ou un groupe d'employés en ce qu'elle leur impose, en raison d'une caractéristique spéciale de cet employé ou de ce groupe d'employés, des obligations, des peines ou des conditions restrictives non imposées aux autres employés ${ }^{46}$.»

Rejetant l'idée que l'on doive prouver une intention afin de conclure à l'existence de discrimination, la Cour suprême est d'avis que dans le cas de la discrimination par suite d'un effet préjudiciable, l'employeur se doit de prendre des mesures raisonnables afin d'accommoder l'employée, à moins que cela ne cause une contrainte excessive à l'exploitation de son entreprise.

45. Commission ontarienne des droits de la personne (O’Malley) c. Simpsons-Sears Ltd., précit. note 44 , par. 22.

46. Ibid., par. 18 . 
La Cour suprême en arrive à cette conclusion en dépit du fait que celle-ci ne trouve aucun fondement dans la formulation du Code ontarien des droits de la personne, lequel prévoyait l'interdiction d'établir une «distinction injuste contre un employé quelconque quant à une clause ou à une condition d'emploi» pour des raisons, notamment, «de croyance». La Cour indique alors ce qui suit:

«[...] il incombe à l'employeur de tenter raisonnablement de faire en sorte que les besoins religieux de l'employée soient respectés à moins que cela ne lui cause une contrainte excessive dans la gestion de ses affaires. Rien dans le Code n'appuie expressément cette thèse d'où une lacune dans le Code et la question: Devrait-on combler cette lacune au moyen de ce principe? [... ${ }^{47}$

À mon avis, quant à l'espèce, la réponse se trouve dans le Code ontarien des droits de la personne, dans son objet et ses dispositions générales. Le Code confère le droit de ne pas être victime de discrimination dans son emploi. Même si aucun droit ne saurait être considéré comme absolu, une conséquence naturelle de la reconnaissance d'un droit doit être l'acceptation sociale de l'obligation générale de le respecter et de prendre des mesures raisonnables afin de le protéger. [...] Dans toute société, les droits d'une personne entreront inévitablement en conflit avec les droits d'autrui. Il est alors évident que tous les droits doivent être limités afin de préserver la structure sociale dans laquelle chaque droit peut être protégé sans porter atteinte indûment aux autres. Cela est particulièrement important lorsque des rapports spéciaux existent, en l'espèce les rapports entre employeur et employé. Dans le présent cas, conformément aux dispositions et à l'objet du Code ontarien des droits de la personne, le droit de l'employé exige que l'employeur prenne des mesures d'accommodement raisonnables ${ }^{48}$.»

Bien que l'employeur ait offert un poste occasionnel à l'employée lui permettant de respecter ses croyances, la Cour suprême est d'avis que, devant le préjudice subi par l'employée, l'employeur se devait d'accommoder l'employée de façon raisonnable, c'est-à-dire, en démontrant que des contraintes de nature économique l'empêchait de s'entendre avec la plaignante afin d'aménager son horaire de travail. Le principe d'accommodement raisonnable ne faisait pas en sorte d'annuler la norme de travail mais obligeait l'employeur à offrir un accommodement individualisé à l'employée. 


\subsection{PARADOXE D'UNE INTERPRÉTATION LITTÉRALE D'UN MOYEN DE DÉFENSE}

Paradoxalement, dans un arrêt rendu le même jour que l'arrêt SimpsonsSears, la Cour suprême du Canada, dans Bhinder, s'appuie sur une formulation différente de la loi en cause, en l'occurrence la loi canadienne sur les droits de la personne ${ }^{49}$, afin d'écarter l'application du principe d'accommodement raisonnable. Dans cette affaire, un employé sikh alléguait une discrimination fondée sur la religion parce qu'il avait été congédié après avoir refusé de porter un casque de sécurité au travail alors que sa religion lui interdisant de porter autre chose qu'un turban sur sa tête.

La Cour est d'avis que le moyen de défense portant sur l'exigence professionnelle prévue à l'article 4 a) de la loi canadienne permet de distinguer ${ }^{50}$ le contexte législatif de l'affaire Bindher de celui prévalant dans l'affaire Simpsons-Sears. Bien que la Cour convienne qu'il s'agit de faits similaires, la formulation de la disposition ${ }^{51}$ en cause dans Bhinder permet expressément à un employeur de poser des conditions de travail ou d'embauche fondées sur des exigences professionnelles justifiées.

49. La loi prévoyait ceci: «14. Ne constituent pas des actes discriminatoires: a) les refus, exclusions, expulsions, suspensions, restrictions, conditions ou préférences de l'employeur qui démontre qu’ils sont fondés sur des exigences professionnelles normales; " [souligné par nous].

50. Bhinder c. CN, [1985] 2 RCS 561, 1985 CSC 19, par. 46: «L'obligation d'accommodement est l'obligation, imposée à l'employeur, de prendre des mesures raisonnables, sans que cela ne cause une contrainte excessive, pour composer avec les pratiques religieuses de l'employé qui est victime de discrimination en raison d'une règle ou d'une condition de travail. Le moyen de défense fondé sur l'exigence professionnelle normale, énoncé à l'al. 14 a), ne laisse aucune place à une obligation de ce genre car il ressort clairement de cet alinéa que, lorsqu'il existe une exigence professionnelle normale, il n'y a pas d'acte discriminatoire. Selon sa formulation dans la Loi canadienne sur les droits de la personne, le moyen de défense fondé sur l'exigence professionnelle normale, lorsqu'il est établi, exclut toute obligation d'accommodement. » [souligné par nous].

51. La Cour adopte une interprétation littérale et grammaticale. La disposition en cause prévoyait que ne sont pas des actes discriminatoires, ceux qui se justifient par «des exigences professionnelles normales $»$. La disposition en cause dans Simpsons-Sears ne contenait pas une telle exception: «4 (1) Nul [...] g) n'établira de distinction injuste contre un employé quelconque quant à une clause ou à une condition d'emploi, pour des raisons de race, de croyance $[\ldots] »$. 


\subsection{INTERPRÉTATION RESTRICTIVE D'UN MOYEN DE DÉFENSE ET CRITÈRES APPLICABLES À LA CONTRAINTE EXCESSIVE}

Quelques années plus tard, en 1990, l'interprétation large du moyen de défense portant sur l'exigence professionnelle justifiée a été renversée dans l'arrêt Central Alberta Dairy Pool ${ }^{52}$, la Cour suprême élargissant ainsi le champ d'application de l'accommodement raisonnable à la discrimination indirecte, même en présence d'une telle défense dans le texte de loi ${ }^{53}$. Dans cette affaire, il était question de l'obligation pour un employé de travailler les jours ouvrables, alors que sa religion ne lui permettait pas de travailler les jours saints et lors du sabbat. Sa demande de congé non payé pour le lundi de Pâques a été refusée en raison des impératifs de l'exploitation de l'entreprise. L'employé a été congédié après qu'il ne se soit pas présenté à son travail le lundi. L'employeur l'avait préalablement informé des conséquences de son absence. Dans sa décision, la Cour suprême donne raison à l'employé en indiquant que l'employeur n'a pas démontré qu'il avait fait les efforts nécessaires pour accommoder celui-ci. Elle mentionne ce qui suit quant à la contrainte excessive:

«C'est à l'employeur qu'incombe le fardeau de prouver qu'il s'est efforcé de tenir compte des croyances religieuses du plaignant, sans s'imposer de contrainte excessive.

Il n'est pas nécessaire de définir de façon exhaustive ce qu'il faut entendre par contrainte excessive. On peut cependant énumérer certains facteurs permettant de la mesurer. Ils peuvent comprendre le coût financier, l'atteinte à la convention collective, le moral du personnel et l'interchangeabilité des effectifs et des installations. L'importance de l'exploitation de l'employeur peut jouer sur l'évaluation de ce qui représente un coût excessif ou sur la facilité avec laquelle les effectifs et les installations peuvent s'adapter au gré des circonstances. Lorsque la sécurité est en jeu, l'ampleur du risque et l'identité de ceux qui le supportent sont des facteurs pertinents. Cette énumération ne se veut pas exhaustive et les résultats qu'on obtiendra en mesurant ces facteurs par rapport au droit de l'employé de ne pas faire l'objet de discrimination varieront nécessairement selon le cas $^{54}$.»

52. Central Alberta Dairy Pool c. Alberta (Commission des droits de la personne), [1990] 2 RCS 489, 1990 CSC 76.

53. L'article 7 de la loi de l'Alberta, Individual's Rights Protection Act, prévoyait une protection contre la discrimination dans l'emploi assortie d'une exception: «(3) Le paragraphe (1) ne s'applique pas à l'égard d'un refus, d'une restriction, d'une condition ou d'une préférence fondée sur une qualification professionnelle normale.» [souligné par nous].

54. Central Alberta Dairy Pool c. Alberta (Commission des droits de la personne), précit. note 52, p. $520-521$. 
Encore une fois, dans une affaire similaire relative à des demandes de congés en raison d'obligations religieuses, la Cour suprême du Canada, dans l'arrêt Renaud ${ }^{55}$, devait se prononcer cette fois-ci sur la question de savoir si l'effet d'une convention collective est pertinent pour évaluer le degré de contrainte résultant des conséquences de mesures d'accommodement sur le respect de la convention collective. Il s'agissait également de déterminer dans quelle mesure l'obligation d'accommodement incombe aussi au syndicat, puisqu'une plainte avait été déposée tant contre l'employeur que contre le syndicat. Le litige reposait sur l'application de la Human Rights Act de la Colombie-Britannique.

Dans ses motifs, la Cour suprême du Canada établit d'abord que la responsabilité du syndicat pourra être engagée de deux façons ${ }^{56}$. En premier lieu, le syndicat peut avoir contribué à la discrimination en participant à l'élaboration de la norme discriminatoire contenue dans la convention collective. En deuxième lieu, le syndicat peut être tenu à l'obligation d'accommodement raisonnable lorsque sa collaboration est requise à la mise en œuvre de celui-ci. Dans le premier cas, le syndicat aura l'obligation de rechercher un accommodement raisonnable; dans le deuxième $\operatorname{cas}^{57}$, son obligation ne naîtra que lorsque sa collaboration est requise afin de mettre en ouvre l'accommodement. Dans les deux cas, la contrainte excessive consistera dans le préjudice que l'accommodement envisagé pourrait causer à d'autres employés. En l'espèce, la Cour conclut que tant l'employeur que le syndicat sont responsables de la discrimination et qu'ils ne se sont pas acquittés de leur obligation d'accommodement. La Cour rejette l'argument de contrainte excessive voulant qu'un accommodement ait pu miner le moral des employés: «l'atteinte ou l'inconvénient minimes sont le prix à payer pour la liberté de religion dans une société multiculturelle ${ }^{58} »$. La Cour indique que:

«Le dossier soumis à la Cour ne révèle aucune preuve que les droits d'autres employés auraient vraisemblablement été lésés si on avait composé avec l'appelant. Son affectation à un quart particulier aurait peut-être entraîné un remaniement de l'horaire d'autres employés, mais il aurait pu être fait avec le consentement de l'employé ou des employés en question. Apparemment, les intimés n’ont pas étudié à fond cette possibilité. Le syndicat s'est opposé à la mesure d'accommodement proposée pour le motif que l'intégrité de la convention collective serait

55. Central Okanagan School District No. 23 c. Renaud, [1992] 2 RCS 970, 1992 CSC 81.

56. Ibid., p. 990.

57. Ibid., p. 993.

58. Ibid., p. 985. 
compromise et non pas en raison de l'opposition d'un employé fondée sur une atteinte à son droit ${ }^{59}$.»

En matière de contrainte excessive, la Cour suprême indique que s'il faut «tenir compte de l'opposition des employés qui résulte de craintes légitimes que leurs droits soient lésés [...] les oppositions fondées sur des attitudes incompatibles avec les droits de la personne ne sont pas pertinentes ${ }^{60} »$. La Cour conclut que le syndicat avait l'obligation initiale d'accommodement. Toutefois, en insérant dans la convention collective l'horaire de travail; en insistant pour qu'il soit respecté; et en refusant la mesure d'accommodement proposée par l'employeur, celui-ci a contribué à la discrimination.

En 1994, la Cour suprême du Canada, dans l'affaire Commission scolaire régionale de Chambly ${ }^{61}$, devait se pencher sur la question de savoir si le jour de congé autorisé par l'employeur en raison d'une fête religieuse de trois enseignants de religion juive devait être rémunéré. Il s'agissait d'un litige fondé sur les dispositions législatives de la Charte québécoise, notamment, les articles 3 (liberté de religion), 10 (égalité, sans discrimination) et 16 (discrimination dans les conditions d'emploi).

La Cour suprême conclut qu'en permettant aux enseignants de prendre un jour de congé sans traitement pour célébrer le Yom Kippour, l'employeur ne s'était pas acquitté de son fardeau de démontrer qu'il avait rempli son obligation d'accommodement sans contrainte excessive. Aucune preuve n'avait été présentée par l'employeur que payer les enseignants de religion juive absents le jour d'une fête religieuse lui imposerait un fardeau financier déraisonnable.

Reprenant les facteurs énumérés dans l'arrêt Renaud, la Cour ajoute ce qui suit:

«Ces facteurs ne sont pas coulés dans le béton. Il y a lieu de les appliquer d'une manière souple et conforme au bon sens, en fonction des faits de chaque cas. Les situations changeront sans cesse. Par exemple, dans une grande entreprise, il peut être relativement facile de remplacer un employé par un autre. Cependant, dans une petite entreprise, le remplacement d'un employé peut imposer un fardeau déraisonnable ou inacceptable à l'employeur. Les conséquences financières d'une mesure d'accommodement varieront aussi indéfiniment. Ce qui peut être parfaitement raisonnable en période de prospérité est susceptible d'imposer

59. Ibid., p. $988-989$

60. Ibid., p. 988.

61. Commission scolaire régionale de Chambly c. Bergevin, [1994] 2 RCS 525, 1994 CSC 102. 
à un employeur un fardeau financier déraisonnable en période de restrictions budgétaires ou de récession. Cependant, les facteurs énumérés peuvent servir de point de départ pour examiner ce qui peut constituer une mesure d'accommodement raisonnable.

Il importe de se rappeler que l'obligation d'accommodement est limitée par les mots "raisonnable" et "sans s'imposer de contrainte excessive". Il s'agit là non pas de critères indépendants, mais plutôt de différentes façons d'exprimer le même concept ${ }^{62}$.»

\subsection{UNE NOUVELLE APPROCHE CONCEPTUELLE, PLUS ENGLOBANTE, DE L'ACCOMMODEMENT RAISONNABLE}

En 1999, dans l'arrêt « Meiorin » ${ }^{63}$, la Cour suprême du Canada apportait un nouvel élargissement à l'application du principe d'accommodement raisonnable en prévoyant que cette obligation doit être prévue dans la norme elle-même. En d'autres mots, pour être jugée raisonnablement nécessaire, la norme « doit composer avec les différences individuelles dans la mesure où cela ne cause aucune contrainte excessive ${ }^{64} »$. La Cour adoptait ainsi une approche plus englobante du droit à l'égalité sans discrimination. La Cour indique en citant les auteurs Day et Brodsky que l'accommodement pris en tant qu'exception à la norme "permet à ceux qui se considèrent "normaux" de continuer à établir des institutions et des rapports à leur image, pourvu qu'ils "composent" avec ceux qui en contestent l'établissement ${ }^{65}$ ».

Nous commentions ainsi ce changement de posture conceptuelle de la part de la Cour suprême du Canada:

«D'autre part, l'arrêt Meiorin opère un véritable changement de paradigme par l'incorporation de l'obligation d'accommodement dans la norme elle-même. Considérant que l'accommodement individuel, par essence, exalte et conforte nécessairement l'assimilation à la norme dominante, l'arrêt Meiorin consacre au plan conceptuel une autre étape dans la compréhension du phénomène discriminatoire, du moins sur le plan théorique, en ce qu'elle conceptualise la norme de façon plus englobante ${ }^{66}$.»

62. Central Okanagan School District No. 23 c. Renaud, précit. note 55, p. 546.

63. Colombie-Britannique (Public Service Employee Relations Commission) c. BCGSEU, [1999] 3 RCS 3, 1999 CSC 652.

64. Ibid., par. 55 .

65. Ibid., par. 41

66. M. Montpetit et $\mathrm{S}$. Bernatchez, «La valse-hésitation du droit à l'égalité pour le bal des dix ans de la Cour McLachlin», Revue nationale de droit constitutionnel, vol. 26, $\mathrm{n}^{\circ} 2$, 2010 , p. 250. 
Même si l'énoncé de principe établi par la Cour suprême du Canada dans l'arrêt Meiorin ne portait pas sur un cas de discrimination en matière religieuse $^{67}$, ce nouveau positionnement quant à la conceptualisation du droit à l'égalité sans discrimination et de l'obligation d'accommodement raisonnable est applicable en toute matière et à l'ensemble de la législation sur les droits de la personne.

Une fois la preuve d'une discrimination à première vue établie, la méthode d'analyse applicable en matière d'accommodement raisonnable, en vigueur depuis 1999, consiste en ce qui suit:

«L'employeur peut justifier la norme contestée en établissant selon la prépondérance des probabilités:

(1) qu'il a adopté la norme dans un but rationnellement lié à l'exécution du travail en cause;

(2) qu'il a adopté la norme particulière en croyant sincèrement qu'elle était nécessaire pour réaliser ce but légitime lié au travail;

(3) que la norme est raisonnablement nécessaire pour réaliser ce but légitime lié au travail. Pour prouver que la norme est raisonnablement nécessaire, il faut démontrer qu'il est impossible de composer avec les employés qui ont les mêmes caractéristiques que le demandeur sans que l'employeur subisse une contrainte excessive ${ }^{68}$."

C'est cette approche qui a été suivie par la jurisprudence ultérieure jusqu'à ce jour. Ces énoncés sont applicables à toutes les lois sur les droits de la personne au Canada, sauf dans les cas où une disposition prévoit un régime qui permet de justifier la discrimination de façon particulière ${ }^{69}$.

67. Il était question d'une norme relative à la condition physique d'une pompière qui ne satisfaisait pas à la norme aérobique adoptée par l'employeur.

68. Colombie-Britannique (Public Service Employee Relations Commission) c. BCGSEU, [1999] 3 RCS 3, 1999 CSC 652, par. 54.

69. À titre d'illustration, V. Nouveau-Brunswick (Commission des droits de la personne) c. Potash Corporation of Saskatchewan Inc., 2008 CSC 45. Dans cet arrêt, la Cour suprême limite l'application des arrêts Grismer et Meiorin. V. commentaires in M. MontPETIT et S. BERNATCHEZ, art. précit. note 66: «La juge Abella, au nom des juges majoritaires, estime que lorsque la loi fournit un régime particulier qui permet de justifier la discrimination, le texte de loi doit prévaloir, rejetant du coup l'argument invoqué par la Commission des droits de la personne du Nouveau-Brunswick selon lequel les critères établis dans les arrêts Meiorin et, surtout, Grismer devraient s'appliquer à l'exception prévue pour les régimes de pension et de retraite.» 


\section{LES RÉPONSES DU POLITIQUE ET DU LÉGISLATIF À LA PROBLÉMATIQUE DES ACCOMMODEMENTS RELIGIEUX}

Dans les années 2000, à la suite de certains jugements de la Cour suprême du Canada, relatifs à la construction d'une souccah sur les balcons d'une copropriété d'habitation et au port du kirpan à l'école publique par un jeune sikh de douze ans, un débat sociétal a eu cours au Québec sur la question des accommodements raisonnables. Plus de vingt ans après que la Cour suprême a énoncé pour la première fois la notion d'accommodement raisonnable, voilà que son usage dans certains contextes religieux a semblé attirer l'attention des journalistes et susciter l'intérêt d'une partie de la population. De nombreux arguments ont été avancés pour critiquer les accommodements raisonnables en matière religieuse.

Certaines interprétations judiciaires, comme le fait de permettre à un jeune de douze ans de porter le kirpan pour aller à l'école publique, ont été considérées comme contraires aux principes du libéralisme, lesquels consacrent notamment la liberté individuelle et l'autonomie de la volonté ${ }^{70}$. Selon la philosophie libérale, seuls les intérêts fondamentaux font l'objet d'une protection constitutionnelle. Or, en interprétant très largement les droits et libertés pour y inclure des intérêts certes importants, mais qui ne répondent pas au critère des intérêts fondamentaux, qui doivent être vitaux, les tribunaux accordent une reconnaissance constitutionnelle à certaines pratiques de manière à leur conférer une priorité normative, ce qui heurte les fondements mêmes de la thèse du libéralisme.

Les affaires Amselem ${ }^{71}$ et Multani ${ }^{72}$, qui sont à l'origine de ces remises en cause, émanaient toutes deux du Québec, plus précisément de l'île de Montréal. Paradoxalement ces deux arrêts ont été associés à la notion d'accommodement raisonnable alors que ni l'un ni l'autre n'emprunte la logique juridique relative à cette notion. La Cour suprême a plutôt tranché ces affaires sur la base de la liberté de religion, alors que le raisonnement juridique aurait pu être tenu en vertu du droit à l'égalité et de la protection contre la discrimination. Par exemple, dans Amselem, la Cour aurait pu fonder son jugement sur les articles 10 et 13 de la Charte québécoise, lesquels combinés protègent contre la discrimination dans un acte juridique (en l'espèce, une déclaration

70. L. B. TRemblay, « Le principe de proportionnalité dans une société démocratique égalitaire, pluraliste et multiculturelle», R.D. McGill, 57, 2012, p. 429.

71. Syndicat Northcrest c. Amselem, précit. note 20.

72. Multani c. Commission scolaire Marguerite-Bourgeoys, précit. note 34. 
de copropriété). Plus encore, l'affaire Multani a été décidée sur la base de la liberté de religion de la Charte canadienne, plutôt que sur le droit à l'égalité de la Charte québécoise, ce qui a amené la majorité de la Cour suprême du Canada à confondre le test de la limitation des droits sous l'article premier de la Charte canadienne avec la logique de l'accommodement raisonnable qui sied moins bien à la restriction émanant d'une règle de droit générale ${ }^{73}$.

Face au tollé provoqué par certains cas d'accommodements raisonnables, le gouvernement du Québec a créé, en 2007, la «Commission de consultation sur les pratiques d'accommodement reliées aux différences culturelles », coprésidée par l'historien et sociologue Gérard Bouchard et le philosophe Charles Taylor. La Commission Bouchard-Taylor a été chargée de dresser un portrait fidèle des pratiques d'accommodement reliées aux différences culturelles et d'effectuer une analyse des enjeux qui y sont associés. Dans son rapport final, la Commission a considéré qu'il ne s'agissait que d'une crise des perceptions, qui traduisait un malaise identitaire ${ }^{74}$.

La Commission n'a pas orienté sa réflexion vers les aspects juridiques de l'accommodement religieux, estimant que l'encadrement légal était suffisamment bien balisé par les règles et les principes existants. Déjà, le nom et le mandat de la Commission ne suggéraient pas que le problème était d'ordre juridique alors qu'il émanait bel et bien de cette discipline. Cela explique peut-être que, dans l'interprétation du mandat qui lui a été confié, la Commission ne se soit pas concentrée, pour l'essentiel, sur les tenants et aboutissants de l'accommodement raisonnable comme rouage juridique ${ }^{75}$. Une telle étude aurait néanmoins permis de traiter du problème fondamental que posaient les accommodements religieux, soit l'acceptation sociale du droit, en vertu de laquelle les justiciables reconnaissent la validité et la légitimité d'une norme juridique et acceptent de modifier leurs comportements et leurs actions pour rendre cette norme effective ${ }^{76}$.

73. Comme l'a reconnu ultérieurement la Cour dans Alberta c. Hutterian Brethren of Wilson Colony, précit. note 25 .

74. Commission de consultation sur les pratiques d'accommodement reliées aux différences culturelles, Fonder l'avenir. Le temps de la conciliation, Rapport, coprésid. G. BOUCHARD et Ch. TAYlor, 2008, p. 18.

75. Commission de consultation sur les pratiques d'accommodement reliées aux différences culturelles, Accommodements et différences. Vers un terrain d'entente: la parole aux citoyens, Document de consultation, coprésid. G. Bouchard et Ch. TAYlor, 2007, p. 20.

76. S. Bernatchez, «Un rapport au droit difficile. La commission Bouchard-Taylor et les accommodements raisonnables», in INSTITUT CANADIEN D'ÉTUDES JURIDIQUES SUPÉRIEURES, Les Journées strasbourgeoises 2008, Droits de la personne. Éthique et droit: nouveaux défis, 
Dans son rapport final, la Commission a même proposé d'écarter la notion juridique d'accommodement raisonnable, pour lui substituer celle d'ajustement concerté ${ }^{77}$. Alors qu'elle limite l'accommodement raisonnable à la voie judiciaire, elle associe l'ajustement concerté à la négociation et la recherche de compromis. Favorisant cette dernière voie, qu'elle qualifie de citoyenne, la Commission n'a pas fait le lien entre la notion juridique d'accommodement raisonnable et cette voie citoyenne, creusant ainsi davantage l'écart, ou le fossé, entre le droit et le monde vécu ${ }^{78}$.

Dans ses recommandations, le rapport final préconisait que le port de signes religieux soit interdit aux magistrats, aux procureurs de la Couronne, aux policiers, aux gardiens de prison, aux président et vice-présidents de l'Assemblée nationale, mais autorisé aux enseignants, aux fonctionnaires, aux professionnels de la santé et à tous les autres agents de l'État. Différents projets de réforme législative ont été envisagés pour donner suite au rapport Bouchard-Taylor sur ce point, sans qu'ils aboutissent toutefois. Ce n'est qu'en octobre 2017 que l'Assemblée nationale du Québec a adopté la loi favorisant le respect de la neutralité religieuse de l'État et visant notamment à encadrer les demandes d'accommodements pour un motif religieux dans certains organismes ${ }^{79}$. En plus d'affirmer la neutralité religieuse de l'État, cette loi impose notamment aux membres du personnel des organismes publics le devoir de neutralité religieuse dans l'exercice de leurs fonctions. Elle vise de plus à reconnaître l'importance d'avoir le visage découvert lorsque des services publics sont donnés et reçus, et ce, afin de s'assurer de la qualité des communications entre les personnes et de permettre la vérification de l'identité de celles-ci, ou pour des fins de sécurité. Enfin, la loi prévoit en outre des critères devant être pris en considération dans le traitement des demandes d'accommodement pour un motif religieux découlant de l'application de la Charte des droits et libertés de la personne.

C'est la première fois que la législation québécoise énonce de tels critères à considérer pour l'encadrement juridique des demandes d'accommodement, cette question ayant été auparavant laissée aux développements de la jurisprudence. Pour l'essentiel, la loi vient reformuler et codifier les critères jurisprudentiels, en plus d'affirmer le principe de l'égalité entre les femmes et les hommes. L'article 11 de la loi dispose:

Cowansville, Y. Blais, 2009, p. 69; S. BernATCHEZ, «Les enjeux juridiques du débat québécois sur les accommodements raisonnables», R.D.U.S., 38, 2007, p. 233.

77. Commission de consultation sur les pratiques d'accommodement reliées aux différences culturelles, Fonder l'avenir. Le temps de la conciliation, op. cit., p. 19.

78. S. BeRnAtChez, «Un rapport au droit difficile...», art. cit., p. 69.

79. Ch. R-26.2.01. 
«Lors du traitement d'une demande d'accommodement pour un motif religieux résultant de l'application de l'article 10 de la Charte des droits et libertés de la personne (chapitre C-12), l'organisme s'assure:

$1^{\circ}$ que la demande est sérieuse;

$2^{\circ}$ que l'accommodement demandé respecte le droit à l'égalité entre les femmes et les hommes ainsi que le droit de toute personne d'être traitée sans discrimination;

$3^{\circ}$ que l'accommodement demandé respecte le principe de la neutralité religieuse de l'État;

$4^{\circ}$ que l'accommodement est raisonnable, c'est-à-dire qu'il ne doit imposer aucune contrainte excessive eu égard, entre autres, au respect des droits d'autrui, à la santé ou à la sécurité des personnes, au bon fonctionnement de l'organisme, ainsi qu'aux coûts qui s'y rattachent. Un accommodement ne peut être accordé que si le demandeur a collaboré à la recherche d'une solution qui satisfait au caractère raisonnable. »

À l'article 12 de la loi, on peut lire que «Le ministre établit des lignes directrices portant sur le traitement d'une demande d'accommodement pour un motif religieux afin d'accompagner les organismes dans l'application de l'article 11 de la présente loi.»

Dès l'élaboration de la loi et lors des travaux préparatoires, la ministre de la Justice de l'époque, Stéphanie Vallée, a déclaré que le gouvernement émettrait des lignes directrices une fois la loi adoptée afin de guider l'évaluation des demandes d'accommodement raisonnable ${ }^{80}$. Depuis, les lignes directrices ont été élaborées et rendues publiques ${ }^{81}$. Toutefois, les lignes directrices n'ont été émises que plusieurs mois après l'entrée en vigueur de la loi, et ne sont entrées en vigueur que le 9 mai 2018. Ainsi, dans une décision rendue en décembre 2017, le juge Babak Barin avait suspendu l'application de l'article 10 de la loi concernant les services à visage découvert jusqu'à ce que les lignes directrices soient adoptées:

«If the legislature considers that accommodation provisions are necessary for the application of a law, then it must ensure that these provisions and any accompanying guidelines identified in that law come into force and are available to the public at the same time. In a statute

80. Étude détaillée du projet de loi $\mathrm{n}^{\circ}$ 62, Loi favorisant le respect de la neutralité religieuse de l'État et visant notamment à encadrer les demandes d'accommodements religieux dans certains organismes, $17 \mathrm{~h} 40$ ( $\mathrm{M}^{\mathrm{me}}$ Vallée) : Journal des débats de la Commission des institutions, $1^{\text {re }}$ sess., $41^{\mathrm{e}}$ légis., 20 sept. 2017.

81. Ministère De LA Justice (QuéBeC), Lignes directrices portant sur le traitement d'une demande d'accommodement pour un motif religieux: https://www.justice.gouv.qc.ca/fileadmin/user_ upload/contenu/documents/Fr_ffrancais_/centredoc/publications/ministere/dossiers/ neutralite/PL62-lignes-FR.pdf [consulté le 6 févr. 2018] 
such as the Act, there is perhaps as much public interest in section 11 as there may be in section 10. These two sections must operate hand in hand if the intention of the legislature is to offer accommodation to those who may be seeking it in the face of the government's actions ${ }^{82}$.»

Ainsi, pour que l'article 11 de la loi puisse trouver application, il est nécessaire de consulter les lignes directrices, qui ont donc été publiées postérieurement à l'entrée en vigueur de la loi. De ce fait, les demandes qui ont été déposées entre l'entrée en vigueur de la loi et l'adoption des lignes directrices n'ont pu être analysées qu'une fois ces dernières adoptées. La ministre aurait pu inclure les critères énumérés dans les lignes directrices directement à l'intérieur du texte de la loi, mais elle a choisi de ne pas le faire et de créer un autre type de norme. De plus, ces lignes directrices ne sont en fait qu'une codification des critères jurisprudentiels pour l'évaluation d'une demande d'accommodement raisonnable pour des motifs religieux.

Finalement, un deuxième jugement ${ }^{83}$ a été rendu par le juge Blanchard concernant les articles 10 et 17 de la loi et la section des lignes directrices concernant le cas d'une demande concernant l'obligation d'avoir le visage découvert ${ }^{84}$. Expliquant qu'il sera très difficile d'appliquer l'article 17 à certains services, puisqu'il oblige les organismes visés à désigner, au sein de leur personnel, un répondant en matière d'accommodement ${ }^{85}$ alors que les services pourront être rendus par plusieurs employés, le juge écrit: «[...] it remains unclear, to say the least, as to how a person will be accommodated on an individual basis, for example to be able to ride the bus, knowing that the accommodation is personal in nature and that the person will be in contact with a great number of different persons delivering the services in question ${ }^{86}$. »

82. National Council of Canadian Muslims (NCCM) c. Attorney General of Quebec, 2017 QCCS 5459, par. 53.

83. National Council of Canadian Muslims (NCCM) c. Attorney General of Québec, 2018 QCCS 2766.

84. Lignes directrices portant sur le traitement d'une demande d'accommodement pour un motif religieux, p. 13.

85. L'article 17 dispose: «Il appartient à la personne qui exerce la plus haute autorité administrative sur les membres du personnel visés aux chapitres II et III de prendre les moyens nécessaires pour assurer le respect des mesures qui y sont prévues. À cette fin, elle doit notamment désigner, au sein de son personnel, un répondant en matière d'accommodement.

Ce répondant a pour fonctions de conseiller la plus haute autorité administrative ainsi que les membres du personnel de l'organisme en matière d'accommodement et de leur formuler des recommandations ou des avis dans le cadre du traitement des demandes reçues. »

86. National Council of Canadian Muslims (NCCM) c. Attorney General of Québec, 2018, précit. note 83 , par. 13. 
Ainsi, l'application de l'article 10 de la loi, en raison des difficultés que posent les articles 11 et 17, ainsi que les lignes directrices en matière de services à visage découvert, a été suspendue.

Les élections générales du $1^{\text {er }}$ octobre 2018 ont porté au pouvoir un nouveau gouvernement, dirigé par le Premier ministre François Legault, chef de la Coalition avenir Québec. Ce gouvernement projette d'adopter une loi sur la laïcité et les signes religieux. Selon le programme du parti politique, il est prévu que le port de signes religieux visibles soit interdit aux employés de l'État qui se trouvent en situation d'autorité. Outre les postes déjà prévus dans la recommandation de la commission Bouchard-Taylor, le gouvernement souhaite élargir l'interdiction aux enseignants dans les écoles publiques.

\section{CONCLUSION}

Le principe juridique d'accommodement raisonnable en matière religieuse a été développé à partir de la reconnaissance des phénomènes et des mécanismes discriminatoires qui font obstacle à la mise en œuvre réelle et effective du droit à l'égalité. Plus particulièrement en matière religieuse, les tribunaux ont reconnu que les pratiques et les normes en apparence neutres peuvent néanmoins être préjudiciables lorsqu'elles ont un effet d'exclusion. C'est à partir de ces constats que les tribunaux ont élargi la conceptualisation du droit à l'égalité afin d'y intégrer non seulement la possibilité d'un traitement différentiel en matière de pratiques religieuses, mais aussi la façon de repenser les normes de façon à ce qu'elles soient plus inclusives.

Le débat sur les accommodements en matière religieuse a suscité à plusieurs reprises la controverse. Bien que la jurisprudence ait contribué à une meilleure compréhension des enjeux et à une transformation de la société, une «crise des perceptions» a mené au Québec à des interventions de nature politique et législative afin de tenter d'assurer l'acceptation sociale du droit - en vertu de laquelle les justiciables reconnaissent la validité et la légitimité d'une norme juridique et acceptent, en conséquence, de modifier leurs comportements et leurs actions pour rendre cette norme effective. Bien que rien ne soit achevé en matière d'accommodement religieux, il demeure que c'est la jurisprudence développée par les tribunaux en matière de droit à l'égalité qui demeure à ce jour l'élément phare d'une société inclusive. 